\title{
NEW MEAN VALUES FOR HOMOGENEOUS SPATIAL TESSELLATIONS THAT ARE STABLE UNDER ITERATION
}

\author{
Christoph ThäLE ${ }^{1}$ AND Viola Weiss ${ }^{2}$ \\ ${ }^{1}$ Département de Mathématiques, Chemin du Musée 23, Université de Fribourg, CH-1700 Fribourg, Suisse; \\ ${ }^{2}$ Fachbereich Grundlagenwissenschaften, Carl-Zeiss-Promenade 2, Fachhochschule Jena, D-07745 Jena, \\ Deutschland \\ e-mail: christoph.thaele@unifr.ch, viola.weiss@ fh-jena.de \\ (Accepted July 27, 2010)
}

\begin{abstract}
Homogeneous random tessellations in the 3-dimensional Euclidean space are considered that are stable under iteration - STIT tessellations. A classification of vertices, segments and flats is introduced and a couple of new metric and topological mean values for them and for the typical cell are calculated. They are illustrated by two examples, the isotropic and the cuboid case. Several extremum problems for these mean values are solved with the help of techniques from convex geometry by introducing an associated zonoid for STIT tessellations.
\end{abstract}

Keywords: convex geometry, iteration/nesting, mean values, random tessellation, spatial statistics, stochastic geometry, zonoid.

\section{INTRODUCTION}

A central problem in stochastic geometry is the development of mathematical models for random structures whose properties are mathematically feasible and not only accessible by simulation. A standard example, where this account is fulfilled is the so-called Boolean model, (see Stoyan et al., 1995; Schneider and Weil, 2008). Mathematically well developed models for random tessellations are Poisson line or plane tessellations and PoissonVoronoi tessellations. Since random tessellations can successfully be applied in material sciences, geology or biology (Stoyan et al., 1995), our problem mentioned at the beginning arises especially in the theory of random tessellations. Particular structures modeled by random tessellations are single-phase polyhedral microstructures, foams, systems of cracks (joints, fissures) in rocks, craquelée of thin layers or systems of cells.

However, the two mathematically manageable standard models for random tessellations, the Poisson line or plane and the Poisson-Voronoi tessellation, seem not in any case to serve the best choices for idealized mathematical models regarding the above mentioned examples. This is mainly due to the fact that their cells are face-to-face (see the definition below). Simulations of random tessellations which are not face-to-face are shown in Figs. 1 and 4, whereas in contrast Fig. 3 shows a realization of a face-to-face tessellation in $\mathbb{R}^{3}$.

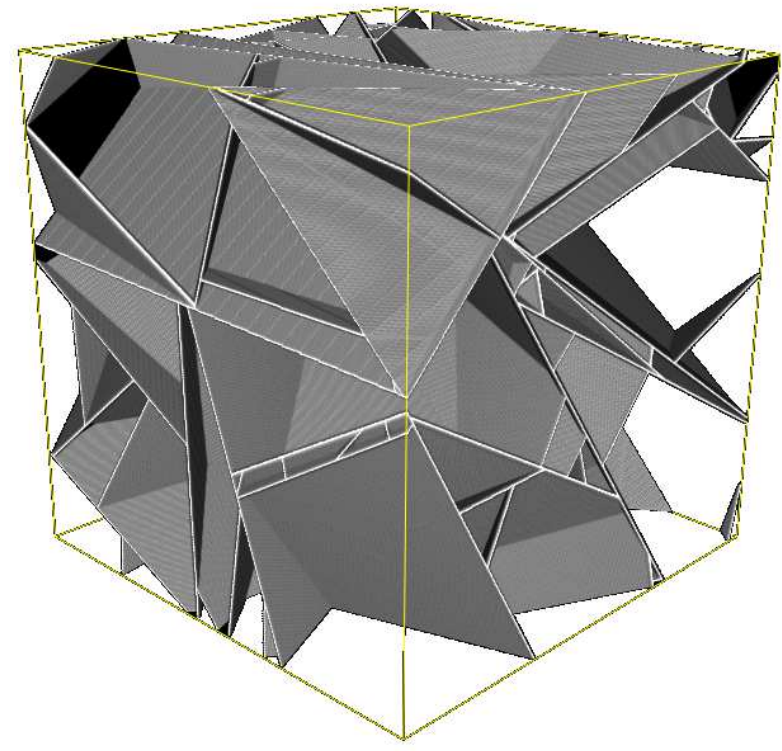

Fig. 1. A realization of a homogeneous and isotropic STIT tessellation.

The underlying models used here for the nonface-to-face cases are random tessellations which are stable under the operation of iteration - so-called STIT tessellations. They were formally introduced by Nagel and Weiss (2005). In later papers Nagel and Weiss (2006; 2008) have shown that many mean values can be obtained from the characteristic stability property of the tessellations by writing and solving certain balance equations. Because of that, random STIT tessellations have the potential to serve as a 
new mathematical reference model - besides Poisson line or plane and Poisson-Voronoi tessellations - for random structures tessellating the plane or the space.

It is the aim of the present paper to continue the work of Nagel and Weiss (2006) and especially that of Nagel and Weiss (2008) by calculating further mean values for random STIT tessellations in the 3-dimensional Euclidean space. We will generalize the planar concept of so-called $I-, J$ - and $K$ segments introduced in Mackisack and Miles (1996) by considering in the spatial case four types of segments and three different types of facets, called flats here. Moreover, we will distinguish between two types of vertices, the T-type and X-type vertices. It is one of the main purposes of the present paper to find mean values which allow a topological characterization of the different types of vertices, segments and flats. Because STIT tessellations are not face-to-face there are interesting multifaceted new effects, for example it appears that the interior of a cell-facet contains vertices and edges. We will explore some of these effects below.

Another class of problems the paper deals with concerns extrema for the considered mean values. STIT tessellations are characterized by their surface intensity and by their so-called directional distribution and the metric mean values depend on both parameters. For a fixed surface intensity it is interesting to ask for which choices of directional distributions these mean values become minimal. Similar questions were studied in the literature for Poisson line, plane or even hyperplane tessellations before, see Schneider and Weil (2008). The answer to these questions can be given with the help of an associated convex body - the Steiner compact - and two inequalities known from convex geometry.

The paper is structured as follows: After a short introduction on spatial random tessellations we rephrase the definition of STIT tessellations and recall some of their main features which are frequently used later. Afterwards, we introduce the different types of vertices, segments and flats appearing in spatial STIT tessellations. Subsequently their mean values are calculated, summarized for clearness and specialized for two particular examples. Extremum problems for the metric mean values will be discussed at the end of the paper by introducing an associated zonoid for STIT tessellations.

\section{SPATIAL RANDOM TESSELLATIONS AND BASIC NOTATION}

A tessellation of the 3-dimensional Euclidean space is a countable and locally finite family of convex polytopes, the cells of the tessellation. They cover the whole space and have pairwise no common interior points. The primitive elements of a tessellation are the vertices, edges, plates and cells. The edges are line segments with no vertices in their relative interior and the plates are convex polygons with no vertices and edges in their relative interior. The boundary of a plate consists of vertices and edges. With a spatial tessellation we can associate a family of $k$-networks for $k=0,1,2$, where a $k$-network is the union of the primitive elements of dimension $k$ of the tessellation. We denote by $V, E, P, C$ the class of vertices, edges, plates and cells of a tessellation, respectively. An object of a class $X$ is often referred to as "an object of type $X ", X \in\{V, E, P, C\}$.

Our definition does not exclude that on the boundary of a plate for example there could appear additional vertices (i.e., elements from the 0-network) which are no corners of the polygon. This is the case when the cells of the tessellation are not face-to-face, see Schneider and Weil (2008, p. 447) for a formal definition and Fig. 2 for an illustration.

To avoid terminological confusion and to distinguish between the primitive elements and the boundary structure of polygons and polytopes, we call the 0 -faces of a polygon or a polytope its corners. The 1-faces of a polygon are its sides and the 1-faces of a polytope are called its ridges. Furthermore, we call the 2-dimensional faces of a spatial polytope its facets.

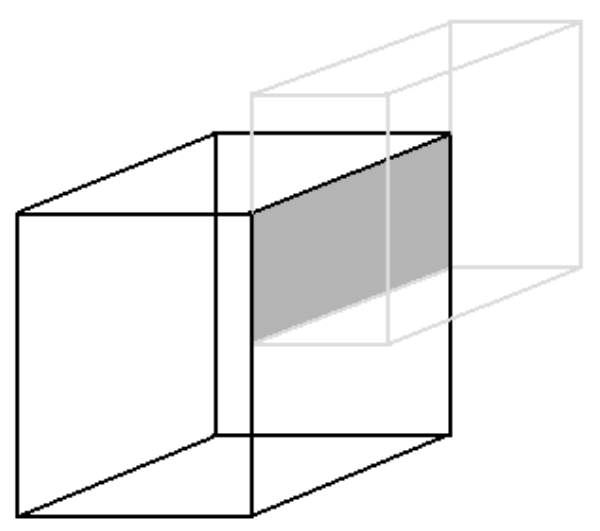

Fig. 2. Cells that are not face-to-face. 
Spatial random tessellations were formally introduced in Schneider and Weil (2008) or Stoyan et al. (1995). They can be seen as random variables with values in the measurable space of spatial tessellations. Similarly to the case of deterministic tessellations we can consider also for random tessellations the collection of cells (formally the 3-network) and the 0-, 1- and 2-network. In this paper we will only consider homogeneous random tessellations, whereby we require the distribution of the tessellation to be invariant under the group of all translations in $\mathbb{R}^{3}$. Moreover, a random tessellation is said to be isotropic, if its distribution is invariant under the group $S O(3)$ of all rotations in $\mathbb{R}^{3}$. In the homogeneous (and not necessarily isotropic case) the technique of Palm distributions allows us to speak of the typical vertex, edge, plate or cell of a random tessellation. For example the typical vertex can be interpreted as a vertex 'uniformly' chosen from the 0 -network in a very large observation window (this can be made precise in the usual sense of ergodic theory by considering the uniform distribution on the set of vertices in a large observation window and by letting the diameter of this window tend to infinity together with a suitable renormalization). Whenever in this paper the word typical appears, it refers to such a definition. For the mathematically exact theory we cite again Schneider and Weil (2008) or Stoyan et al. (1995).

To define mean values for typical objects of a random tessellation, we have to formalize in advance the concept of adjacent objects. An object $x$ of type $X$ is said to be adjacent to an object $y$ of type $Y$ if either $x \subseteq y$ or $y \subseteq x$. Let $N_{X, Y}$ be the mean number of objects of type $Y$ adjacent to the typical object of class $X$, where the expectation is taken with respect to the Palm distribution of objects of type $X$. On the other hand, the primitive elements of dimension $k, k=0,1,2,3$, are $k$-dimensional polytopes with $j$-dimensional polytopal faces, $j<k$. For the typical primitive element of type $X$ of dimension $k$ we denote by $v_{j}(X)$ the mean number of its $j$-faces, $j<k$. For example we have attentively to distinguish between the mean number of vertices of the typical cell $N_{C, V}$ and the mean number $v_{0}(C)$ of its corners. For tessellations in face-to-face position that differentiation is not necessary, since both parameters coincide.

By $\lambda_{X}$ we denote the intensity of objects of class $X$, by which is meant the intensity of the homogeneous point-process of centroids of $X$-type objects (where the centroid function is assumed to be invariant under translations). This is the mean number of centroids of objects of class $X$ per unit volume, which by homogeneity is a well defined quantity and does not depend on the choices of 'the unit volume' and the centroid function.

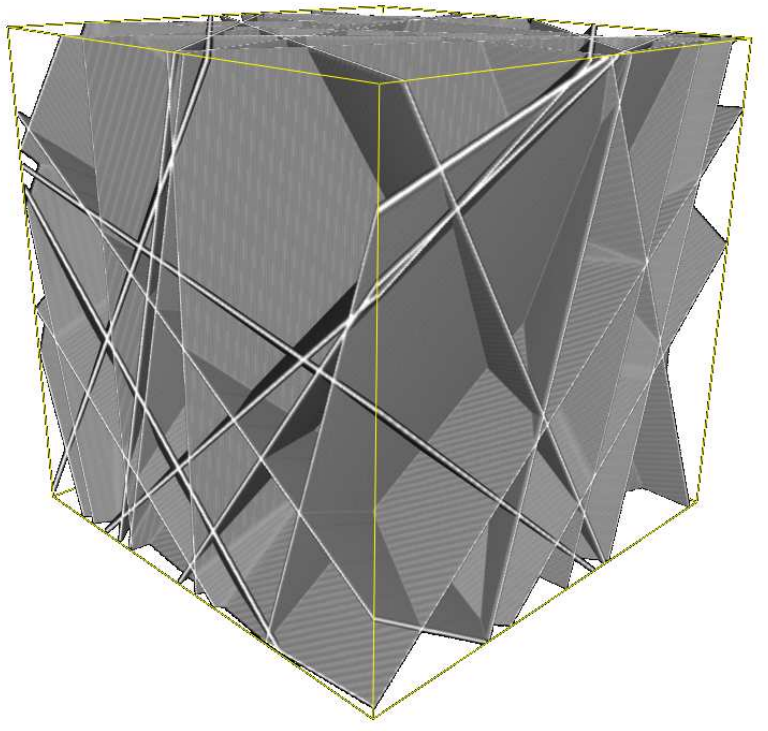

Fig. 3. A realization of a homogeneous and isotropic Poisson plane tessellation.

As an example of a homogeneous random spatial tessellation we consider the homogeneous Poisson plane tessellation, which is a plane tessellation induced by a homogeneous Poisson process on the space of planes in $\mathbb{R}^{3}$. A realization of a homogeneous and isotropic Poisson plane tessellation is shown in Fig. 3. It is well known that the law of a Poisson plane tessellation is uniquely determined by a positive real constant $\tau$, its intensity, and a non-degenerate probability measure $\mathcal{R}$ on the upper unit half-sphere (Schneider and Weil, 2008, and especially Chapter 10.3 therein). Such a random collection of planes decomposes the space into convex polytopes whose interiors are pairwise disjoint. It is a face-to-face tessellation as can easily be seen from the definition. For the typical cell of a Poisson plane tessellation, usually called the Poisson polytope, the following mean values are well known, see Stoyan et al. (1995):

$$
\begin{aligned}
& v_{0}(C)=N_{C, V}=8, \quad v_{1}(C)=N_{C, E}=12, \\
& v_{2}(C)=N_{C, P}=6,
\end{aligned}
$$

i.e., the Poisson polytope has in the mean 8 vertices, 12 edges and 6 plates and likewise 8 corners and 12 ridges and 6 facets. These values are frequently used in the course of the paper.

\section{STIT TESSELLATIONS}

STIT tessellations form an interesting class of homogeneous random tessellations, whose cells are 
not in a face-to-face position and whose properties are mathematically feasible. They formally arise as limits of rescaled iterations (or nestings) of homogeneous random tessellations. The idea of iteration is to subdivide the cells of a given homogeneous tessellation independently by a sequence of independent and identically distributed homogeneous random tessellations having the same distribution as the primary tessellation. An appropriate rescaling is necessary in order to keep the surface intensity $S_{V}$, i.e., the mean total surface area of cell boundaries per unit volume, constant. STIT tessellations can be characterized by the property that their distribution does not change through rescaled iteration, i.e., we require the distribution of the tessellation to be stable under iteration, a property which also explains the abbreviation STIT. A realization of a homogeneous and isotropic random STIT tessellation is shown in Fig. 1, whereas Fig. 4 shows a realization of a homogeneous but anisotropic STIT tessellation in the 3-dimensional space. In Nagel and Weiss (2005) an explicit construction was presented for such tessellations in a bounded convex window in Euclidean spaces of arbitrary dimension $d \geq 2$.

The finite-volume construction can be understood as a process of sequential cell division at random times. At time $t=0$ the construction starts with a compact convex polytopal window $W \subset \mathbb{R}^{3}$, for example a cube or a ball. After a random lifetime $t_{1}$ that is exponentially distributed (with parameter related to the geometry of $W$ and the directional distribution $\mathcal{R})$ a random plane with directional distribution $\mathcal{R}$ is introduced in $W$, thus $W$ splits into two new polytopes and cell-facets (at their birth time also plates) are born with birth time $t_{1}$. Then, sequentially, all extant polytopes $W_{1}, \ldots, W_{k}$ with the respective birth times $t_{1}, \ldots, t_{k}$ are divided independently of each other in the same way. The lifetime of $W_{i}$ is a random variable, exponentially distributed and with parameter depending on the geometry of $W_{i}$ and the previously fixed directional distribution $\mathcal{R}$ (when constructing an isotropic tessellation, this lifetime is proportional to the mean breadth of the respective cell). At the end of its lifetime, the polytope $W_{i}$ is subdivided by a random plane, which is chopped of by the boundary of the cell, $W_{i}$ dies and two new polytopes and cell-facets are born. The state at a fixed time $t>0$ is a tessellation of $W$, in the sequel denoted by $\Phi(t, W)$. It can be shown that $\Phi(t, W)$ is consistent in $W$, which means that it is independent of $W$ and there exists a homogeneous random tessellation $\Phi(t)$ of the whole space $\mathbb{R}^{3}$ such that

$$
\Phi(t, W) \stackrel{D}{=} \Phi(t) \cap W
$$

with $\stackrel{D}{=}$ standing for equality in distributions. Remarkably, the tessellations $\Phi(t, W)$ and $\Phi(t)$ enjoy the stability property with respect to (rescaled) iteration.

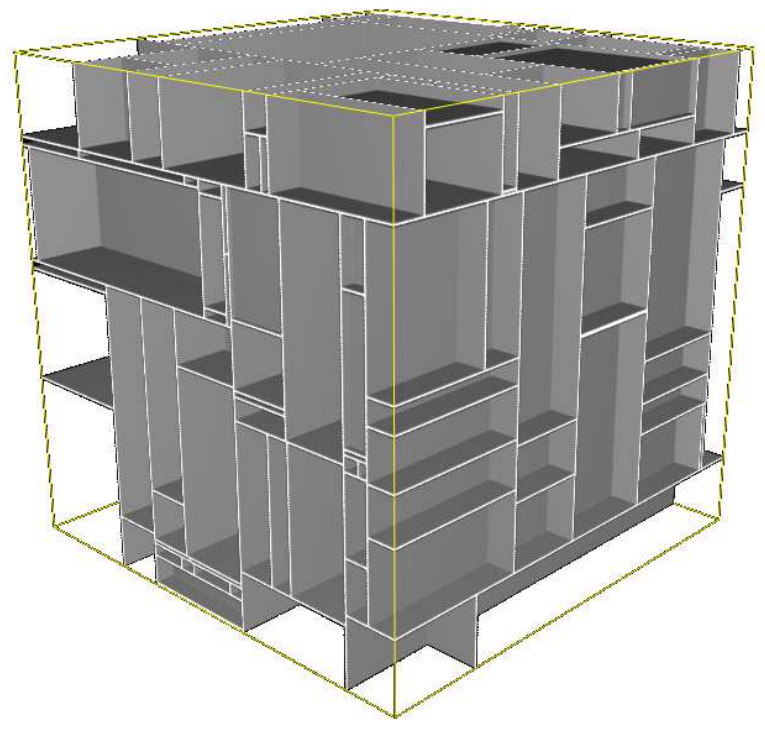

Fig. 4. A realization of a homogeneous and anisotropic STIT tessellation whose directional distribution is concentrated with equal weight to the three coordinate directions.

The algorithmic construction of STIT tessellations in polytopal windows described above immediately leads to an effective simulation algorithm. This algorithm was used to create the simulations shown in Figs. 1 and 4.

A direct global construction of a whole-space STIT tessellations was found in Mecke et al. (2008a) and Mecke et al. (2008b). Some mean values for homogeneous random STIT tessellations in the plane appeared in Nagel and Weiss (2006) and for the spatial case in Nagel and Weiss (2008). Some of the therein obtained formulas will be recalled in a later section.

We will now list some of the key-properties of STIT tessellations, which are important for the present paper. We will formulate them for the 3dimensional case, even if they are valid for arbitrary space dimensions:

- The law of a homogeneous random STIT tessellation in $\mathbb{R}^{3}$ is uniquely determined by its surface intensity $0<S_{V}<\infty$ and by a probability measure $\mathcal{R}$ on the upper half-sphere $\mathbb{S}_{+}^{2}$, whose support is not concentrated on a great half-subsphere. The measure $\mathcal{R}$ describes the distribution of the direction of the unit normal 
vector at a uniformly chosen point (the so-called typical point with respect to the boundary measure of cells) of the 2-network associated with the tessellation and $S_{\mathcal{V}}$ is the mean total surface area of cell boundaries per unit volume.

- The interior of the typical cell of a spatial homogeneous random STIT tessellation has the same distribution as the interior of the Poisson polytope with parameters $S_{\mathcal{V}}$ and $\mathcal{R}$. This means that mean volume, mean surface area and length of the edge skeleton of the typical cell of a STIT tessellation are the same as for the Poisson polytope. The difference between STIT and Poisson plane tessellations arises from the mutual arrangement of the cells (compare Figs. 1 and 4 with Fig. 3) which implies that the topological parameters, such as the mean number of vertices, edges and plates adjacent to the typical cell, will differ.

- The cells of STIT tessellations are not face-to-face, which means that the intersection of two cells with a common plate is not necessarily a cell-facet of both cells. An example of two cells that are not face-to-face is shown in Fig. 2.

- The intersection of a homogeneous STIT tessellation in $\mathbb{R}^{3}$ with a plane induces a homogeneous random STIT tessellation in this intersection plane. The induced random tessellation will be isotropic, whenever the spatial tessellation is isotropic, too. If we intersect a homogeneous random STIT tessellation in $\mathbb{R}^{3}$ with a line $g$, we obtain a homogeneous Poisson point process on $g$.

For details on STIT tessellations we refer to Nagel and Weiss (2005), Nagel and Weiss (2006), Mecke et al. (2007), Nagel and Weiss (2008), Mecke et al. (2008a) and Mecke et al. (2008b), Mecke (2009), Thäle (2009) or Schreiber and Thäle (2010). Also Schneider and Weil (2008), pp. 469-470 contains some comments on STIT tessellations.

In Weiss and Cowan (paper submitted for publication), a systematic study of spatial homogeneous tessellations which are not face-to-face is undertaken. STIT tessellations are considered there as one particular example and the results for mean values are deduced from rather general formulae. In the current paper, the mean values for STIT tessellations will be derived from the topology and the special properties of these tessellations. Furthermore, Weiss and Cowan do not consider what we later call I-segments and I-flats.

\section{TYPES OF VERTICES}

Homogeneous planar STIT tessellations only have T-shaped vertices, which means that in any vertex there are three outgoing edges and two of them are collinear. From the construction of a STIT tessellation given above it follows that a homogeneous spatial STIT tessellation has two different types of vertices, namely

- T-vertices and

- X-vertices.

A T-vertex appears on a ridge of a cell, when this cell is intersected by a plate which is born, whereas an X-vertex emerges, when two plates born in two neighboring cells intersect in the relative interior of a common plate of the two cells. These two possible situations are illustrated in Fig. 5. Note that the notion of a $\mathrm{T}$ - or X-vertex is based on the plates which create that vertex. The two types of vertices and the geometric differences between them are essential for our further considerations as will be seen below.
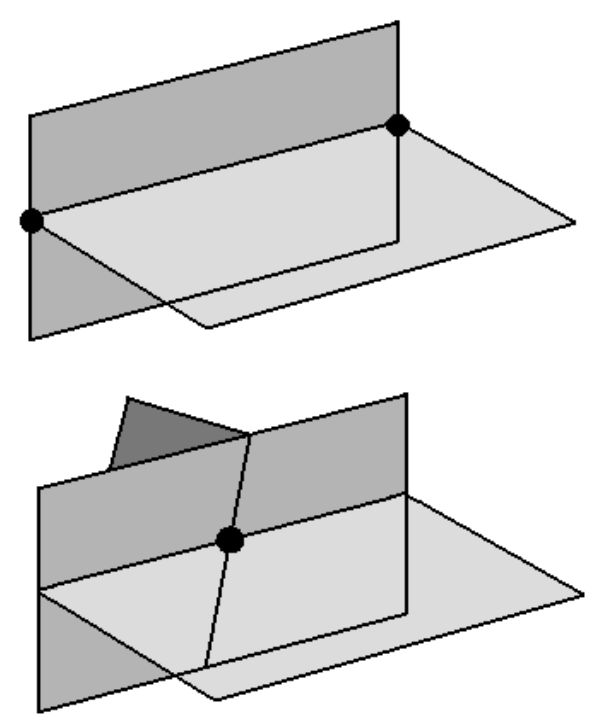

Fig. 5. A T-vertex (top) and an X-vertex (bottom).

The intensities of T- and X-type vertices, i.e., mean number of $\mathrm{T}$ - and $\mathrm{X}$-vertices per unit volume, are denoted by $\lambda_{V_{T}}$ and $\lambda_{V_{X}}$, respectively. Furthermore, $\lambda_{V}=\lambda_{V_{T}}+\lambda_{V_{X}}$ is the intensity of vertices, meaning the mean number of vertices of the tessellation per unit volume. 


\section{TYPES OF SEGMENTS}

For planar tessellations whose cells are not in a face-to-face position, Mackisack and Miles (1996) introduced a classification of the linear segments of the 1-network associated with the tessellation. More precisely, they introduced the notion of $I-, J$ - and $K$-segments for the planar case. For spatial STIT tessellations we introduce now a similar concept and make the following classification for segments contained in the 1-dimensional network of the 3dimensional STIT tessellation:

- a $K$-segment is an edge of the tessellation, that means it is a linear segment in the 1-network between two vertices but with no further vertex in its relative interior,

- an I-segment is the maximal union of connected and collinear $K$-segments, that means it cannot be enlarged by another collinear $K$-segment (alternatively, the $I$-segments are the sides of the plates born during the spatio-temporal construction of STIT tessellations explained earlier),

- a $J^{(2)}$-segment is a side of a plate and

- a $J^{(3)}$-segment is a ridge of a cell, see Fig. 6.

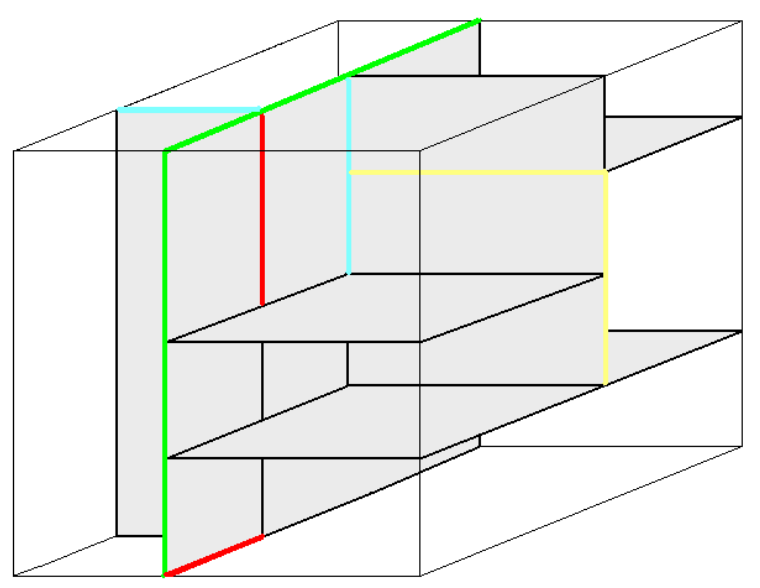

Fig. 6. Each time two $K$-segments (red), $J^{(2)}$-segments (cyan), $J^{(3)}$-segments (yellow) and I-segments (green).

Note that the class of $K$-segments is equal to the class of edges $E$ of the tessellation. The $J$ segments are 1-dimensional faces, i.e., the sides, of the primitive elements of the tessellation, $J^{(2)}$ of the 2-dimensional primitive elements, the plates, and $J^{(3)}$ that of the 3-dimensional primitive elements, the cells. It is important to point out that the point processes formed by the collection of all $J^{(2)}$ - and $J^{(3)}$ segment midpoints, respectively, is not simple, which means that the segments are counted with multiplicity according to the number of plates or cells they belong to.

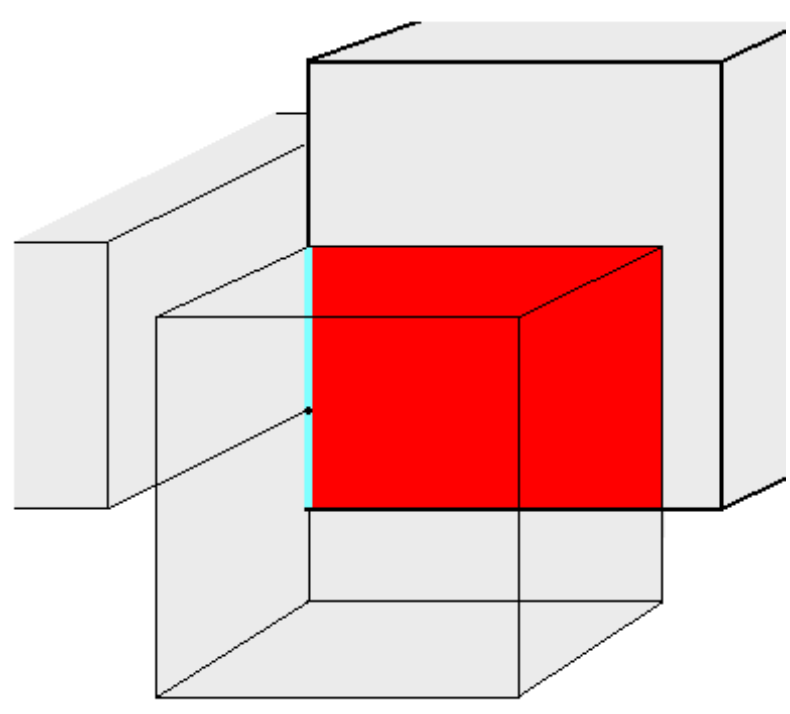

Fig. 7. A plate (red) with a proper $J^{(2)}$-segment (cyan) on its boundary.

In Fig. 7 it is illustrated that the classes $J^{(2)}$ and $J^{(3)}$ do not coincide, by constructing a 'proper' $J^{(2)}$ segment, i.e., one which is not a $K$-, $J^{(3)}$ - or $I$-segment at the same time. To see it, observe at first that the cyan segment in Fig. 7 is a side of the red plate, hence a $J^{(2)}$-segment. Further note, that it has a vertices in its relative interior, hence it is not a $K$-segment, it is also not a ridge of any of the involved cells, thus, it cannot be a $J^{(3)}$-segment. Moreover, it can be enlarged by another collinear $K$-segment, which means that it cannot be an $I$-segment.

The classes of $K-, I-, J^{(2)}$ - and $J^{(3)}$-segments are denoted by $K_{1}, I_{1}, J_{1}^{(2)}$ and $J_{1}^{(3)}$, respectively. For $Y \in$ $\left\{K, I, J^{(2)}, J^{(3)}\right\}$, the intensity of $Y$-segments, i.e., the mean number of $Y$-segment midpoints per unit volume is denoted by $\lambda_{Y_{1}}$. The mean length of the typical $Y$-segment is $L_{Y_{1}}$ and analogously to the concept of adjacent objects, the mean number of vertices in the relative interior of the typical $Y$-segment is denoted by $N_{\text {relint }\left(Y_{1}\right), V}$.

\section{TYPES OF FLATS}

In referring to the different types of segments we consider now the 2-dimensional network associated with a random STIT tessellation and introduce the 
following classification of the different types of plates (we call them flats here in order to distinguish between them, the primitive plates and the faces and facets of the cells):

- a $K$-flat is a plate of the tessellation, that means it is a 2-dimensional convex polygon bounded by edges of the 1-network and without edges and vertices in its relative interior,

- a $J$-flat is a facet of a cell and

- an I-flat is the maximal union of coplanar and connected $K$-flats and cannot be enlarged by another coplanar $K$-flat, see Fig. 8. Alternatively, $I$ flats are the polygons born during the construction of a STIT tessellation homogeneously in time.

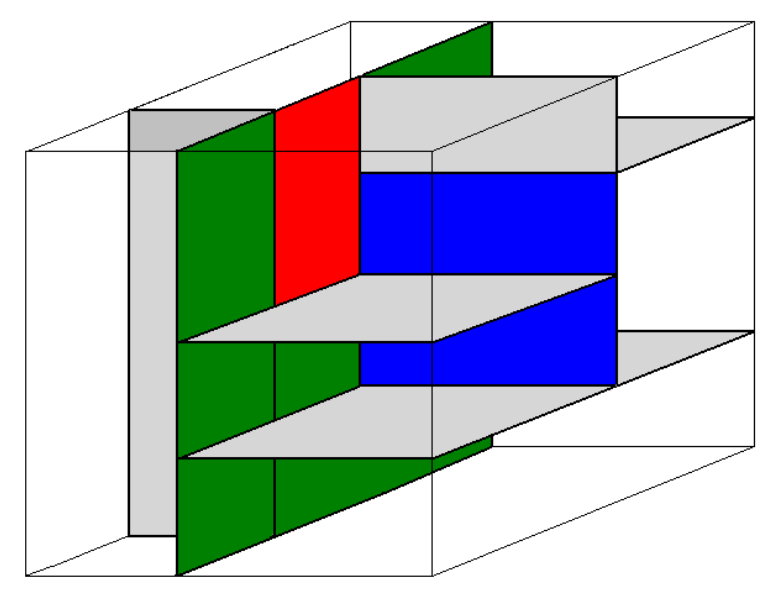

Fig. 8. A K-flat (red), a J-flat (blue) and an I-flat (green).

Note, that by definition the sides of a $K$-flat are $J^{(2)}$-segments, $J^{(3)}$-segments coincide with the sides of $J$-flats and $I$-flats are surrounded by $I$-segments. Moreover, the class of $K$-flats is equal to the class $P$ of plates and the collection of $J$-flats corresponds to the class of cell-facets. Again, the homogeneous point process of $J$-flat centroids is not a simple one in general, since one plate can be a common facet of two neighboring cells.

From now on we denote by $K_{2}, J_{2}$ and $I_{2}$ the classes of $K$-flats, $J$-flats and $I$-flats, respectively, i.e., we use the subscript 2 to make clear the dimension of the objects under consideration in order to distinguish between flats and segments, where for the latter we have used the subscript ${ }_{1}$ already in the last section.

By $\lambda_{X_{2}}$ we denote the intensity of $X$-flats, i.e., the mean number of $X$-flats per unit volume (this is the mean number of $X$-flats centroids per unit volume) and by $A_{X_{2}}$ and $U_{X_{2}}$ the mean area and perimeter of the typical $X$-flat, where $X$ stands for one of the literals $K$, $J$ or $I$. Furthermore, let $N_{\text {relint }\left(X_{2}\right), V}$ and $N_{b d\left(X_{2}\right), V}$ be the mean number of vertices in the relative interior or on the boundary of the typical $X$-flat, respectively.

The mean number of edges in the relative interior or on the boundary of the typical $X$-flat is denoted by $N_{\text {relint }\left(X_{2}\right), E}$ and $N_{b d\left(X_{2}\right), E}$, respectively, with $X \in$ $\{K, J, I\}$, where we say that an edge $e \in E$ is located in the relative interior of an $X$-flat $x \in X$ if $\operatorname{relint}(e) \subseteq$ relint $(x)$. Moreover we introduce $N_{J_{2}, P}$ and $N_{I_{2}, P}$ for the mean number of plates adjacent to the typical $J$ flat or the typical $I$-flat, respectively.

\section{PARAMETERS FOR CELLS}

We will also derive some new topological mean values for the typical cell of a spatial STIT tessellation. As already mentioned in the section on STIT tessellations, the typical cell of a STIT tessellation and the Poisson polytope of a Poisson plane tessellation with the same surface intensity share the same metric mean values, such as mean volume, mean surface area and mean length of the edge skeleton. For this reason we will restrict our attention to the topological parameters. We denote by $s k(c)$ the 1-dimensional boundary, i.e., the edge skeleton of objects $c$ of class C.

We are interested in the following topological mean values: The mean number of vertices $N_{s k}(C), V$ and the mean number of edges $N_{s k(C), E}$ on the edge skeleton of the typical cell.

\section{SUMMARY OF NOTATION}

For clarity we summarize here the notation mostly introduced so far. Let $Y \in\left\{K, J^{(2)}, J^{(3)}, I\right\}$ and $X \in$ $\{K, J, I\}$. Furthermore we use the abbreviations p.u.v. for 'per unit volume', typ. for 'typical' and ri. for relative interior.

\section{Intensities:}

$S_{\mathcal{V}}$ - surface intensity, i.e., mean total surface area p.u.v.

$L_{\mathcal{V}}$ - edge length intensity, i.e., mean total edge length p.u.v.

$\lambda_{V}, \lambda_{E}, \lambda_{P}, \lambda_{C}-$ mean number of vertices, edges, plates, cells p.u.v.

$\lambda_{V_{T}}, \lambda_{V_{X}}-$ mean number of T-, X- vertices p.u.v.

$\lambda_{Y_{1}}, \lambda_{X_{2}}$ - mean number of $Y$-segments and $X$-flats p.u.v.

Typical objects: 
$L_{Y_{1}}-$ mean length of the typ. $Y$-segment

$A_{X_{2}}, U_{X_{2}}-$ mean area and mean perimeter of the typ. $X$-flat

$v_{k}(X), v_{k}(C)-$ mean number of $k$-dimensional polytope face(t)s of the typ. $X$-flat $(k=0,1)$ and the typ. cell $(k=0,1,2)$

\section{Adjacent objects:}

$N_{O_{1}, O_{2}}$ - mean number of primitive objects of type $\mathrm{O}_{2}$ adjacent to the typ. object of type $\mathrm{O}_{1}, \mathrm{O}_{1}, \mathrm{O}_{2} \in$ $\{V, E, P, C\}$ :

$N_{\text {relint }\left(Y_{1}\right), V}-$ mean number of vertices in the r.i. of the typ. $Y$-segment

$N_{\text {relint }\left(X_{2}\right), V}, N_{b d\left(X_{2}\right), V}$ - mean number of vertices in the r.i. and on the boundary of the typ. $X$-flat

$N_{\text {relint }\left(X_{2}\right), E}-$ mean number of edges in the r.i. of the typ. $X$-flat

$N_{J_{2}, P}, N_{I_{2}, P}-$ mean number of plates adjacent to the typ. $J$-flat or to the typ. I-flat, respectively

$N_{s k(C), V}, N_{s k(C), E}-$ mean number of vertices or edges, respectively, in the edge skeleton of the typ. cell

The following mean value relations can easily be derived:

$N_{Y_{1}, V}=N_{\text {relint }\left(Y_{1}\right), V}+2 \quad$ and $\quad N_{Y_{1}, E}=N_{\text {relint }\left(Y_{1}\right), V}+1$

for the mean number of vertices and edges in the relative interior of the typical $Y$-segment,

$$
N_{b d\left(X_{2}\right), E}=N_{b d\left(X_{2}\right), V}
$$

for the mean number of edges on the boundary of the typical $X$-flat,

$$
N_{X_{2}, V}=N_{\text {relint }\left(X_{2}\right), V}+N_{b d\left(X_{2}\right), V}
$$

and

$$
N_{X_{2}, E}=N_{\text {relint }\left(X_{2}\right), E}+N_{b d\left(X_{2}\right), V}
$$

for the mean number of vertices and edges adjacent to the typical $X$-flat.

\section{KNOWN MEAN VALUES}

We regard from now on a homogeneous spatial STIT tessellation $\Phi\left(S_{\mathcal{V}}\right)$ with surface intensity $0<$ $S_{\mathcal{V}}<\infty$ and fixed directional distribution $\mathcal{R}$, which is a probability measure on the upper half-sphere $\mathbb{S}_{+}^{2}$, such that the set of directions in the support of $\mathcal{R}$ spans the whole 3-dimensional space.
We define the two constants $\zeta_{2}$ and $\zeta_{3}$ by

$$
\begin{aligned}
& \zeta_{2}=\iint\left[u_{1}, u_{2}\right] \mathcal{R}\left(\mathrm{d} u_{1}\right) \mathcal{R}\left(\mathrm{d} u_{2}\right), \\
& \zeta_{3}=\iiint\left[u_{1}, u_{2}, u_{3}\right] \mathcal{R}\left(\mathrm{d} u_{1}\right) \mathcal{R}\left(\mathrm{d} u_{2}\right) \mathcal{R}\left(\mathrm{d} u_{3}\right),
\end{aligned}
$$

where the integration is always over $\mathbb{S}_{+}^{2}$ and $\left[u_{1}, u_{2}\right]$ denotes the area of the parallelogram spanned by $u_{1}$ and $u_{2}$ and $\left[u_{1}, u_{2}, u_{3}\right]$ is the volume of the parallelepiped spanned by the vectors $u_{1}, u_{2}$ and $u_{3}$ (in the terminology of Schneider and Weil, 2008, $\left[u_{1}, u_{2}\right]$ and $\left[u_{1}, u_{2}, u_{3}\right]$ are subspace determinants). These two constants reflect the influence of the directional distribution $\mathcal{R}$ on the mean values of the random tessellation.

In the isotropic case these constants have the particular values $\zeta_{2}=\pi / 4$ and $\zeta_{3}=\pi / 8$ as can be concluded from Thm. 4.6.5 in Schneider and Weil (2008).

The following formulas for mean values for homogeneous spatial STIT tessellations were already proved in Nagel and Weiss (2008):

$$
\begin{aligned}
\lambda_{V} & =S_{\mathcal{V}}^{3} \zeta_{3}, & \lambda_{E} & =2 S_{\mathcal{V}}^{3} \zeta_{3}, \\
\lambda_{P} & =\frac{7}{6} S_{\mathcal{V}}^{3} \zeta_{3}, & \lambda_{C} & =\frac{1}{6} S_{V}^{3} \zeta_{3}, \\
L_{E} & =\frac{1}{2 S_{V}} \frac{\zeta_{2}}{\zeta_{3}}, & & \\
U_{P} & =\frac{18}{7 S_{V}} \frac{\zeta_{2}}{\zeta_{3}}, & A_{P} & =\frac{6}{7 S_{V}^{2}} \frac{1}{\zeta_{3}}, \\
N_{P, V} & =N_{P, E}=\frac{36}{7}, & N_{C, V} & =24, \\
N_{C, E} & =36, & N_{C, P} & =14 .
\end{aligned}
$$

Moreover, for the edge length intensity $L_{\mathcal{V}}$ we have

$$
L_{\mathcal{V}}=S_{v}^{2} \zeta_{2}
$$

These values will be used in the sequel to derive further mean value formulas for STIT tessellations in 3D.

For completeness we recall now the values for the metric mean values of the typical cell. Denoting by Vol $_{C}$ the mean volume, by $S_{C}$ the mean surface area, by $U_{C}$ the mean length of the edge-skeleton (perimeter) and by $B_{C}$ the mean breadth of the typical cell we have

$$
\begin{aligned}
\operatorname{Vol}_{C} & =\frac{6}{S_{\mathcal{V}}^{3}} \frac{1}{\zeta_{3}}, \quad S_{C}=\frac{12}{S_{\mathcal{V}}^{2}} \frac{1}{\zeta_{3}}, \\
U_{C} & =\frac{18}{S_{\mathcal{V}}} \frac{\zeta_{2}}{\zeta_{3}}, \quad B_{C}=\frac{3}{2 S_{V}} \frac{\zeta_{2}}{\zeta_{3}} .
\end{aligned}
$$




\section{TOPOLOGY OF VERTICES}

The two different types of vertices and moreover their topology illustrated by Fig. 5 will play a crucial role. For later reference, we summarize now some of their most important properties. We start with the Ttype vertices and note that a T-vertex is ...

... an endpoint of 4 edges,

$\ldots$ an endpoint of $10 J^{(2)}$-segments and located in the relative interior of another $J^{(2)}$-segment,

... an endpoint of $6 J^{(3)}$-segments and located in the relative interior of another $J^{(3)}$-segment,

$\ldots$ an endpoint of $2 I$-segments and located in the relative interior of a third one,

... a corner of 5 plates and located on the boundary (but no corner) of a sixth plate,

... a corner of $6 J$-flats, located on the boundary (but no corner) of two other $J$-flats and in the relative interior of a ninth one,

... a corner of one $I$-flat, located on the boundary (but no corner) of another $I$-flat and in the relative interior of a third one,

... a corner of 2 cells, located on the edge skeleton (but no corner) of a third cell and in the relative interior of a cell-facet of a fourth one.

$\mathrm{X}$-type vertices have the following topological features: An X-vertex is ...

... an endpoint of 4 edges,

$\ldots$ an endpoint of $8 J^{(2)}$-segments and located in the relative interior of another two $J^{(2)}$-segments,

... located in the relative interior of $4 J^{(3)}$-segments,

... located in the relative interior of $2 I$-segments,,

... a corner of 4 plates and located on the boundary

(but no corner) of two further plates,

... located on the boundary (but no corner) of $8 \mathrm{~J}$ flat,

... located on the boundary (but no corner) of $2 I-$ flats and in the relative interior of a third one,

... located on the edge skeleton of 4 cells.

These relationships will from now on be used frequently in the course of our considerations.

\section{NEW MEAN VALUES FOR VERTICES}

In this section we derive at first expressions for the intensities $\lambda_{V_{T}}$ and $\lambda_{V_{X}}$, i.e., the mean number of Tand $\mathrm{X}$-vertices per unit volume, respectively. To do so, recall that the mean total number of vertices per unit volume equals $S_{V}^{3} \zeta_{3}$, i.e., $\lambda_{V}=\lambda_{V_{T}}+\lambda_{V_{X}}=S_{V}^{3} \zeta_{3}$. We observe now that each T-vertex is a corner of exactly 2 cells and an $\mathrm{X}$-vertex cannot be a corner of any cell. With $v_{0}(C)=8$ we have $8 \lambda_{C}=2 \lambda_{V_{T}}$ and with $\lambda_{C}=\frac{1}{6} S_{\mathcal{V}}^{3} \zeta_{3}$ it follows

$$
\lambda_{V_{T}}=4 \lambda_{C}=\frac{2}{3} S_{\mathcal{V}}^{3} \zeta_{3}
$$

and

$$
\lambda_{V_{X}}=\lambda_{V}-\lambda_{V_{T}}=S_{V}^{3} \zeta_{3}-\frac{2}{3} S_{V}^{3} \zeta_{3}=\frac{1}{3} S_{V}^{3} \zeta_{3} .
$$

Thus, for a spatial STIT tessellation we have obviously the proportion

$$
\lambda_{V_{T}}: \lambda_{V_{X}}=2: 1
$$

Moreover, the following topological mean values are easily derived from the topology of vertices in a spatial STIT tessellation:

$$
\begin{aligned}
& N_{V_{T}, E}=N_{V_{X}, E}=4, \\
& N_{V_{T}, P}=N_{V_{X}, P}=6,
\end{aligned}
$$

and

$$
N_{V_{T}, C}=N_{V_{X}, C}=4,
$$

see Fig. 5.

\section{NEW MEAN VALUES FOR TYPICAL SEGMENTS}

The present section is devoted to mean values for the four different types of segments introduced above. It is our aim to calculate their intensities, their mean lengths and the mean number of vertices in their relative interior, which are denoted by $\lambda_{Y_{1}}, L_{Y_{1}}$ and $N_{\text {relint }\left(Y_{1}\right), V}$, respectively, where $Y$ stands for one of the literals from $\left\{K, J^{(2)}, J^{(3)}, I\right\}$.

Recall at first from the section on known mean values that

$$
\lambda_{K_{1}}=\lambda_{E}=2 S_{\mathcal{V}}^{3} \zeta_{3}
$$

and that

$$
L_{K_{1}}=L_{E}=\frac{1}{2 S_{\mathcal{V}}} \frac{\zeta_{2}}{\zeta_{3}}
$$


Moreover, it follows from the definition of $K$-segments that

$$
N_{\text {relint }\left(K_{1}\right), V}=0 \text {. }
$$

We have seen above that each T-vertex is a corner of exactly 5 plates and each $\mathrm{X}$-vertex is a corner of exactly 4 plates. Thus, we have $\lambda_{P} v_{0}(P)=5 \lambda_{V_{T}}+4 \lambda_{V_{X}}$ and therefore the typical $K$-flat has in the mean four corners, i.e.,

$$
v_{0}(P)=v_{0}\left(K_{2}\right)=4 \text {. }
$$

With $\lambda_{P} v_{0}(P)=\lambda_{J_{1}^{(2)}}$ and $\lambda_{J_{1}^{(2)}} L_{J_{1}^{(2)}}=3 L_{\mathcal{V}}-$ recall that each edge is contained in the boundary of exactly 3 edges and that $J$-segments are counted with multiplicity - we obtain now

$$
\lambda_{J_{1}^{(2)}}=\frac{14}{3} S_{\mathcal{V}}^{3} \zeta_{3} \quad \text { and } \quad L_{J_{1}^{(2)}}=\frac{9}{14 S_{\mathcal{V}}} \frac{\zeta_{2}}{\zeta_{3}} .
$$

The typical $K$-flat has in the mean $\frac{36}{7}$ vertices on its boundary, $N_{P, V}=\frac{36}{7}$. Since - in the mean -4 of them are corners of the plate $(K$-flat) we get

$$
N_{\text {relint }\left(J_{1}^{(2)}\right), V}=\frac{\lambda_{P}}{\lambda_{J_{1}^{(2)}}}\left(N_{P, V}-4\right)=\frac{\frac{36}{7}-4}{4}=\frac{2}{7},
$$

i.e., in the mean, the typical $J^{(2)}$-segment contains $\frac{2}{7}$ vertices in its relative interior.

From the property that a STIT tessellation has a Poisson-typical cell we get immediately

$$
L_{J_{1}^{(3)}}=\frac{1}{S_{\mathcal{V}}} \frac{\zeta_{2}}{\zeta_{3}}
$$

from Stoyan et al. (1995) in the isotropic and Schneider and Weil (2008) in the anisotropic case. The fact that each edge is adjacent to two ridges of cells and that $J$-segments are counted with multiplicities implies

$$
\lambda_{J_{1}^{(3)}}=\frac{2 L_{\mathcal{V}}}{L_{J_{1}^{(3)}}}=2 S_{\mathcal{V}}^{3} \zeta_{3}
$$

To obtain $N_{\text {relint }\left(J_{1}^{(3)}\right), V}$ we observe that $L_{J_{1}^{(3)}}=2 L_{K_{1}}$, which yields

$$
N_{\operatorname{relint}\left(J_{1}^{(3)}\right), V}=1
$$

We calculate now the intensity $\lambda_{I_{1}}$, i.e., the mean number of $I$-segments per unit volume. To this end we notice that any T-vertex is the endpoint of exactly two $I$-segments and any $I$-segment has two endpoints and they are T-vertices. Thus $2 \lambda_{I_{1}}=2 \lambda_{V_{T}}$, which leads to

$$
\lambda_{I_{1}}=\frac{2}{3} S_{v}^{3} \zeta_{3}
$$

From the equation $\lambda_{I_{1}} L_{I_{1}}=L_{\mathcal{V}}$ we get furthermore

$$
L_{I_{1}}=\frac{L_{\mathcal{V}}}{\lambda_{I_{1}}}=\frac{3}{2 S_{\mathcal{V}}} \frac{\zeta_{2}}{\zeta_{3}} \text {. }
$$

Moreover, each T-vertex lies in the relative interior of exactly one I-segment and each X-vertex in the relative interior of exactly two. By taking into account the proportion of $\mathrm{T}$ - and $\mathrm{X}$-vertices, we arrive at

$$
N_{\text {relint }\left(I_{1}\right), V} \cdot \lambda_{I_{1}}=1 \cdot \lambda_{V_{T}}+2 \cdot \lambda_{V_{X}}=\frac{4}{3} \lambda_{V}
$$

Hence,

$$
N_{\text {relint }\left(I_{1}\right), V}=\frac{\frac{4}{3} \lambda_{V}}{\lambda_{I_{1}}}=2,
$$

meaning that the typical $I$-segment contains 2 vertices in its relative interior in the mean. Note, that this is in line with the observation that $L_{I_{1}}=3 L_{K_{1}}$.

\section{NEW MEAN VALUES FOR TYPICAL FLATS}

Mean values for the typical $K$-flat were considered by Nagel and Weiss (2008). They obtained

$$
\begin{array}{ll}
\lambda_{K_{2}}=\frac{7}{6} S_{V}^{3} \zeta_{3}, & A_{K_{2}}=\frac{S_{\mathcal{V}}}{\lambda_{K_{2}}}=\frac{6}{7 S_{\mathcal{V}}^{2}} \frac{1}{\zeta_{3}} \\
N_{K_{2}, V}=N_{K_{2}, E}=\frac{36}{7}, & U_{K_{2}}=\frac{18}{7 S_{\mathcal{V}}} \frac{\zeta_{2}}{\zeta_{3}} .
\end{array}
$$

Additionally, in the previous section we have shown that $v_{0}\left(K_{2}\right)=4$. Moreover, from the definition of $K$ flats it follows

$$
N_{\text {relint }\left(K_{2}\right), V}=N_{\text {relint }\left(K_{2}\right), E}=0 .
$$

We consider now the typical $J$-flat. Since STIT tessellations have Poisson typical cells, the mean area of the typical $J$-flat is the same as the mean area of a facet of the Poisson polytope, which is well known from the literature (see Stoyan et al., 1995, for the isotropic case and Schneider and Weil, 2008, p. 490, for the mean value in the anisotropic case). From this it follows

$$
A_{J_{2}}=\frac{2}{S_{V}^{2}} \frac{1}{\zeta_{3}}, \quad U_{J_{2}}=\frac{4}{S_{\mathcal{V}}} \frac{\zeta_{2}}{\zeta_{3}}
$$

and also

$$
v_{0}\left(J_{2}\right)=4,
$$

i.e., the typical $J$-flat is in the mean a topological quadrangle. 
The intensity $\lambda_{J_{2}}$ can be calculated from the relation $\lambda_{J_{2}} A_{J_{2}}=2 S_{V}$, which yields

$$
\lambda_{J_{2}}=S_{\mathcal{V}}^{3} \zeta_{3} \text {. }
$$

To consider the mean number of edges in the relative interior of the typical $J$-flat, we use the following topological property of STIT tessellations: Any edge of the tessellation is adjacent to three cells. In one of them that edge is contained in the relative interior of a facet and in the two others it is contained on a ridge of these cells, in order that we obtain

$$
N_{\text {relint }\left(J_{2}\right), E}=\frac{\lambda_{E}}{\lambda_{J_{2}}}=2 .
$$

Next we remark, that any T-vertex is contained in the relative interior of exactly one $J$-flat and that any Xvertex is contained in the relative interior of no $J$-flat. This implies

$$
N_{\text {relint }\left(J_{2}\right), V}=\frac{\lambda_{V_{T}}}{\lambda_{J_{2}}}=\frac{2}{3} .
$$

Any vertex of a STIT tessellation is located on the boundary of eight cell-facets. Therewith we obtain

$$
N_{b d\left(J_{2}\right), V}=N_{b d\left(J_{2}\right), E}=\frac{8 \lambda_{V}}{\lambda_{J_{2}}}=8 .
$$

The mean number of plates adjacent to the typical $J$ flat is given by

$$
N_{J_{2}, P}=\frac{2 \lambda_{P}}{\lambda_{J_{2}}}=\frac{7}{3},
$$

because each plate is a part of two $J$-flats. Summarizing, we find for homogeneous spatial STIT tessellations the following mean value relation

$$
N_{J_{2}, P} \cdot A_{P}=A_{J_{2}} .
$$

Now, we consider the typical $I$-flat, whose mean area is given by

$$
A_{I_{2}}=\frac{6}{S_{V}^{2}} \frac{1}{\zeta_{3}} .
$$

This can heuristically be seen as follows (the mathematical justification of this approach is provided by Corollary 2 in Schreiber and Thäle, 2010): We regard the STIT tessellation under consideration as a time-augmented random process of I-flats, where the flats are marked by their respective birth times $\beta \in\left(0, S_{\mathcal{V}}\right)$ in referring to the construction explained in the section on STIT tessellations. Observe now, that conditioned on its birth time $0<\beta=s<S_{\mathcal{V}}$, the mean area $A_{I_{2}}(\beta=s)$ of the typical $I$-flat is given by

$$
A_{I_{2}}(\beta=s)=\frac{2}{s^{2}} \frac{1}{\zeta_{3}}
$$

as follows from the fact that STIT tessellations have Poisson typical cells, the observation that sectional STIT tessellations are again stable under iteration and the known mean values for Poisson line and plane tessellations in Stoyan et al. (1995) or Schneider and Weil (2008). In order to obtain the unconditioned mean value $A_{I_{2}}$ we have to integrate $A_{I_{2}}(\beta=s)$ with respect to all possible birth times. To this end we need the birth time distribution of the typical $I$-flat or its density $p_{\beta}(\cdot)$. To obtain a formula, note that from invariance reasons (homogeneity of the tessellation) it follows that the intensity $\lambda_{I_{2}}(\beta<s)$ of $I$-flats with birth time smaller than $s$ equals $c s^{3}$ with some universal constant $c>0$ not depending on $s$. Thus, by the definition of the mark distribution as given in Chap. 3.5 of Schneider and Weil (2008) we see that the birth time distribution is given by

$$
\mathbb{P}(\beta<s)=\frac{\lambda_{I_{2}}(\beta<s)}{\lambda_{I_{2}}\left(\beta<S_{\mathcal{V}}\right)}=\frac{c s^{3}}{c S_{\mathcal{V}}^{3}}=\frac{s^{3}}{S_{\mathcal{V}}^{3}}, 0<s<S_{\mathcal{V}} .
$$

Hence, the birth time density $p_{\beta}(s)$ equals

$$
p_{\beta}(s)=\frac{3 s^{2}}{S_{\mathcal{V}}^{3}}, 0<s<S_{\mathcal{V}} .
$$

Integration of $A_{I_{2}}(\beta=s)$ with respect to this birth time density yields immediately the value for $A_{I_{2}}$ :

$$
\begin{aligned}
A_{I_{2}} & =\int_{0}^{S_{\mathcal{V}}} A_{I_{2}}(\beta=s) p_{\beta}(s) \mathrm{d} s \\
& =\int_{0}^{S_{\mathcal{V}}} \frac{2}{s^{2}} \frac{1}{\zeta_{3}} \cdot \frac{3 s^{2}}{S_{\mathcal{V}}^{3}} d s=\frac{6}{S_{V}^{2}} \frac{1}{\zeta_{3}} .
\end{aligned}
$$

For a closely related approach in the planar case see Mecke et al. (2010).

Having calculated the mean area of the typical $I$ flat, we observe now that $\lambda_{I_{2}} A_{I_{2}}=S_{V}$ implies

$$
\lambda_{I_{2}}=\frac{S_{\mathcal{V}}}{A_{I_{2}}}=\frac{1}{6} S_{\mathcal{V}}^{3} \zeta_{3} .
$$

From $L_{V}=\lambda_{I_{2}} U_{I_{2}}$ we can calculate the mean perimeter of $I_{2}$, namely

$$
U_{I_{2}}=\frac{L_{V}}{\lambda_{I_{2}}}=\frac{6}{S_{\mathcal{V}}} \frac{\zeta_{2}}{\zeta_{3}} .
$$

Above we have seen that any T-vertex is a corner of exactly one $I$-flat, is located on the boundary but no corner of another $I$-flat, and is contained in the interior of a third one. On the other hand, any X-vertex is located on the boundary and is no corner of two $I$-flats 
and in the interior of a third $I$-flat. We obtain from these considerations

$$
\begin{array}{rlrl}
N_{\text {relint }\left(I_{2}\right), V} & =\frac{\lambda_{V}}{\lambda_{I_{2}}} & & =6, \\
N_{b d\left(I_{2}\right), V} & =\frac{2 \lambda_{V}}{\lambda_{I_{2}}} & & =12, \\
N_{I_{2}, V} & =N_{\text {relint }\left(I_{2}\right), V}+N_{b d\left(I_{2}\right), V} & =18, \\
v_{0}\left(I_{2}\right) & =\frac{\lambda_{V_{T}}}{\lambda_{I_{2}}} & & =4 .
\end{array}
$$

In particular, the typical $I$-flat is in the mean a topological quadrangle, which is not surprising, since $I$-flats are $J$-flats at their birth time.

Any edge of a STIT tessellation is located in the relative interior of exactly one $I$-flat, so that the mean number of edges in the relative interior of the typical I-flat can be calculated from

$$
N_{\text {relint }\left(I_{2}\right), E}=\frac{\lambda_{E}}{\lambda_{I_{2}}}=12 .
$$

For the mean number of plates adjacent to the typical I-flat we obtain

$$
N_{I_{2}, P}=\frac{\lambda_{P}}{\lambda_{I_{2}}}=7 .
$$

From this we get a mean value relation for the typical I-flat of a STIT tessellation analogously to that for the typical $J$-flat:

$$
N_{I_{2}, P} \cdot A_{P}=A_{I_{2}}
$$

\section{NEW MEAN VALUES FOR THE TYPICAL CELL}

To deduce mean values and mean value relations for the typical cell, we consider again the topology of the vertices mentioned earlier in a separate section. Recall, that each T-vertex is located on the edgeskeleton of three cells, whereas each $\mathrm{X}$-vertex is adjacent to the skeleton of four cells. Hence, it follows

$$
N_{s k(C), V}=\frac{3 \lambda_{V_{T}}}{\lambda_{C}}+\frac{4 \lambda_{V_{X}}}{\lambda_{C}}=20 .
$$

The typical cell of a STIT tessellation has in the mean 24 vertices, 20 of them are on the skeleton (8 corners and 12 in the relative interiors of the ridges) and 4 vertices are located in the relative interiors of the cellfacets, in the mean.

Any edge of a STIT tessellation is adjacent to the edge skeleton of two cells, which implies

$$
N_{s k(C), E}=\frac{2 \lambda_{E}}{\lambda_{C}}=24 \text {. }
$$

We conclude that the typical cell of a STIT tessellation has in the mean 36 edges, 24 of them are located on the edge skeleton and 12 in the relative interiors of cellfacets, in the mean.

\section{SUMMARY OF THE MEAN VALUES}

The following tables summarize the mean values obtained so far. First, the new mean values for the typical cell are presented (Table 1). Next, we summarize the new mean values for the different types of vertices (Table 2), segments (Table 3) and flats (Table 4)

Table 1. Topological mean values for the typical cell.

\begin{tabular}{cccl}
\hline$W$ & $C$ & $s k(C)$ & \\
\hline$N_{W, V}$ & 24 & 20 & $v_{0}(C)=8$ \\
$N_{W, E}$ & 36 & 24 & $v_{1}(C)=12$ \\
\hline
\end{tabular}

Table 2. Mean values for vertices.

\begin{tabular}{cccc}
\hline$Z$ & $Z=T$ & $Z=X$ & both \\
\hline$\lambda_{V_{Z}}$ & $\frac{2}{3} S_{\mathcal{V}}^{3} \zeta_{3}$ & $\frac{1}{3} S_{\mathcal{V}}^{3} \zeta_{3}$ & $S_{\mathcal{V}}^{3} \zeta_{3}$ \\
$N_{V_{Z}, E}$ & 4 & 4 & - \\
$N_{V_{Z}, P}$ & 6 & 6 & - \\
$N_{V_{Z}, C}$ & 4 & 4 & - \\
\hline
\end{tabular}

Table 3. Mean values for segments.

\begin{tabular}{ccccc}
\hline$Y_{1}$ & $K_{1}$ & $J_{1}^{(2)}$ & $J_{1}^{(3)}$ & $I_{1}$ \\
\hline$\lambda_{Y_{1}}$ & $2 S_{\mathcal{V}}^{3} \zeta_{3}$ & $\frac{14}{3} S_{\mathcal{V}}^{3} \zeta_{3}$ & $2 S_{\mathcal{V}}^{3} \zeta_{3}$ & $\frac{2}{3} S_{\mathcal{V}}^{3} \zeta_{3}$ \\
$L_{Y_{1}}$ & $\frac{1}{2 S_{\mathcal{V}}} \frac{\zeta_{2}}{\zeta_{3}}$ & $\frac{9}{14 S_{\mathcal{V}}} \frac{\zeta_{2}}{\zeta_{3}}$ & $\frac{1}{S_{\mathcal{V}}} \frac{\zeta_{2}}{\zeta_{3}}$ & $\frac{3}{2 S_{\mathcal{V}}} \frac{\zeta_{2}}{\zeta_{3}}$ \\
$N_{\text {relint }\left(Y_{1}\right), V}$ & 0 & $\frac{2}{7}$ & 1 & 2 \\
$N_{Y_{1}, V}$ & 2 & $\frac{16}{7}$ & 3 & 4 \\
$N_{\text {relint }\left(Y_{1}\right), E}$ & 1 & $\frac{9}{7}$ & 2 & 3 \\
\hline
\end{tabular}


Table 4. Mean values for flats.

\begin{tabular}{cccc}
\hline$X_{2}$ & $K_{2}$ & $J_{2}$ & $I_{2}$ \\
\hline$\lambda_{X_{2}}$ & $\frac{7}{6} S_{\mathcal{V}}^{3} \zeta_{3}$ & $S_{\mathcal{V}}^{3} \zeta_{3}$ & $\frac{1}{6} S_{\mathcal{V}}^{3} \zeta_{3}$ \\
$A_{X_{2}}$ & $\frac{6}{7 S_{\mathcal{V}}^{2}} \frac{1}{\zeta_{3}}$ & $\frac{2}{S_{\mathcal{V}}^{2}} \frac{1}{\zeta_{3}}$ & $\frac{6}{S_{\mathcal{V}}^{2}} \frac{1}{\zeta_{3}}$ \\
$U_{X_{2}}$ & $\frac{18}{7 S_{\mathcal{V}}} \frac{\zeta_{2}}{\zeta_{3}}$ & $\frac{4}{S_{\mathcal{V}}} \frac{\zeta_{2}}{\zeta_{3}}$ & $\frac{6}{S_{\mathcal{V}}} \frac{\zeta_{2}}{\zeta_{3}}$ \\
$v_{0}\left(X_{2}\right)$ & 4 & 4 & 4 \\
$N_{\text {relint }\left(X_{2}\right), V}$ & 0 & $\frac{2}{3}$ & 6 \\
$N_{b d\left(X_{2}\right), V}$ & $\frac{36}{7}$ & 8 & 12 \\
$N_{X_{2}, V}$ & $\frac{36}{7}$ & $\frac{26}{3}$ & 18 \\
$N_{\text {relint }\left(X_{2}\right), E}$ & 0 & 2 & 12 \\
$N_{b d\left(X_{2}\right), E}$ & $\frac{36}{7}$ & 8 & 12 \\
$N_{X_{2}, E}$ & $\frac{36}{7}$ & 10 & 24 \\
$N_{X_{2}, P}$ & 0 & $\frac{7}{3}$ & 7 \\
\hline
\end{tabular}

\section{TWO EXAMPLES}

The new metric mean values considered so far, i.e., the quantities $L_{Y_{1}}, U_{X_{2}}$ and $A_{X_{2}}$ with $Y \in$ $\left\{K, J^{(2)}, J^{(3)}, I\right\}$ and $X \in\{I, J, K\}$ are now calculated explicitly in two special situations. Namely, when the directional distribution $\mathcal{R}$ is the uniform distribution on $\mathbb{S}_{+}^{2}-$ the isotropic case, see Fig. 1 - and when $\mathcal{R}$ is concentrated with equal weight on three orthogonal directions. Since in the latter case the cells are cuboids with probability 1 we call this case the cuboid case, see Fig. 4 for an illustration.

Isotropic case: Here we have $\zeta_{2}=\pi / 4$ and $\zeta_{3}=\pi / 8$, which leads to

$$
\begin{array}{ll}
L_{K_{1}}=\frac{1}{S_{\mathcal{V}}}, & L_{J_{1}^{(2)}}=\frac{9}{7 S_{\mathcal{V}}}, \\
L_{J_{1}^{(3)}}=\frac{2}{S_{\mathcal{V}}}, & L_{I_{1}}=\frac{3}{S_{\mathcal{V}}}, \\
U_{K_{2}}=\frac{36}{7 S_{\mathcal{V}}}, & U_{J_{2}}=\frac{8}{S_{\mathcal{V}}},
\end{array}
$$

$$
\begin{array}{ll}
U_{I_{2}}=\frac{12}{S_{\mathcal{V}}}, & A_{K_{2}}=\frac{48}{7 \pi S_{\mathcal{V}}^{2}}, \\
A_{J_{2}}=\frac{16}{\pi S_{\mathcal{V}}^{2}}, & A_{I_{2}}=\frac{48}{\pi S_{\mathcal{V}}^{2}} .
\end{array}
$$

Cuboid case: Here we have $\zeta_{2}=3 ! / 3^{2}=2 / 3$ and $\zeta_{3}=$ $3 ! / 3^{3}=2 / 9$ and hence

$$
\begin{aligned}
L_{K_{1}} & =\frac{3}{2 S_{\mathcal{V}}}, & L_{J_{1}^{(2)}} & =\frac{27}{14 S_{\mathcal{V}}}, \\
L_{J_{1}^{(3)}} & =\frac{3}{S_{\mathcal{V}}}, & L_{I_{1}} & =\frac{9}{2 S_{\mathcal{V}}}, \\
U_{K_{2}} & =\frac{54}{7 S_{\mathcal{V}}}, & U_{J_{2}} & =\frac{12}{S_{\mathcal{V}}} \\
U_{I_{2}} & =\frac{18}{S_{\mathcal{V}}}, & A_{K_{2}} & =\frac{27}{7 S_{\mathcal{V}}^{2}}, \\
A_{J_{2}} & =\frac{9}{S_{\mathcal{V}}^{2}}, & A_{I_{2}} & =\frac{27}{S_{\mathcal{V}}^{2}}
\end{aligned}
$$

\section{SOME EXTREMUM PROBLEMS}

We have seen in the section on STIT tessellations that the law of a homogeneous spatial random STIT tessellation is uniquely characterized by its surface intensity and by its directional distribution. The surface intensity is a positive real constant $0<S_{\mathcal{V}}<\infty$ and the directional distribution $\mathcal{R}$ is a probability measure on the upper half-sphere $\mathbb{S}_{+}^{2}$ fulfilling the non-degeneracy condition from above.

Recall, that a convex body $K \subset \mathbb{R}^{3}$ is uniquely determined by its support function $h(K, \cdot)$ defined by

$$
h(K, u):=\max \{\langle x, u\rangle: x \in K\}, \quad u \in \mathbb{R}^{3} .
$$

For the law of any homogeneous random STIT tessellation with surface intensity $0<S_{\mathcal{V}}<\infty$ and directional distribution $\mathcal{R}$ we define now a convex body $\Pi\left(S_{\mathcal{V}}, \mathcal{R}\right)$ by putting

$$
h\left(\Pi\left(S_{\mathcal{V}}, \mathcal{R}\right), u\right):=S_{\mathcal{V}} \int_{\mathbb{S}_{+}^{2}}|\langle u, v\rangle| \mathcal{R}(\mathrm{d} v), \quad u \in \mathbb{R}^{3} .
$$

The centrally symmetric convex body $\Pi\left(S_{\mathcal{V}}, \mathcal{R}\right)$ is called Steiner compact associated with the law of the STIT tessellation or its associated zonoid, by referring to the characteristic property of $\Pi\left(S_{\mathcal{V}}, \mathcal{R}\right)$. Notice, that the zonoid $\Pi\left(S_{\mathcal{V}}, \mathcal{R}\right)$ is at the same time the associated zonoid of the homogeneous random Poisson plane tessellation with the same parameters, see Schneider and Weil (2008). From the general theory ibidem we infer that $\Pi\left(S_{\mathcal{V}}, \mathcal{R}\right)$ is uniquely determined by the parameters $S_{\mathcal{V}}$ and $\mathcal{R}$ and that 
given a centered zonoid there is exactly one law of a random STIT tessellation having the given convex body as its Steiner compact. Because of this one-toone correspondence all mean values for homogeneous spatial STIT tessellations are expressible in terms of the geometric characteristics of the associated Steiner compact. These are:

$$
\begin{aligned}
& \operatorname{Vol}(\Pi)=\operatorname{Vol}\left(\Pi\left(S_{\mathcal{V}}, \mathcal{R}\right)\right) \text { - the volume of } \\
& \Pi\left(S_{\mathcal{V}}, \mathcal{R}\right), \\
& S f(\Pi)=S f\left(\Pi\left(S_{\mathcal{V}}, \mathcal{R}\right)\right) \text { - the surface area of } \\
& \Pi\left(S_{\mathcal{V}}, \mathcal{R}\right), \\
& \operatorname{br}(\Pi)=\operatorname{br}\left(\Pi\left(S_{\mathcal{V}}, \mathcal{R}\right)\right) \text { - the mean breadth of } \\
& \Pi\left(S_{\mathcal{V}}, \mathcal{R}\right) .
\end{aligned}
$$

Here, we use $V o l, S f$ and $b r$ instead of $V, S$ and $B$ in order to distinguish between these functionals and the mean values considered in an earlier section.

Note that from Thm. 10.3.3 in Schneider and Weil (2008) it follows that $\operatorname{Vol}(\Pi), S f(\Pi)$ and $\operatorname{br}(\Pi)$ are in terms of $S_{\mathcal{V}}, \zeta_{2}$ and $\zeta_{3}$ given by

$$
\begin{aligned}
\operatorname{Vol}(\Pi) & =\frac{S_{\mathcal{V}}^{3}}{6} \zeta_{3}, \\
S f(\Pi) & =S_{\mathcal{V}}^{2} \zeta_{2}, \\
b r(\Pi) & =\frac{S_{\mathcal{V}}}{2} .
\end{aligned}
$$

The metric mean values from the last sections can be expressed in terms of the geometric parameters of П. Since STIT tessellations have Poisson typical cells we can deduce again from Thm. 10.3.3. in Schneider and Weil (2008) that

$$
A_{J_{2}}=\frac{2}{3} \frac{b r(\Pi)}{\operatorname{Vol}(\Pi)}, \quad L_{J_{1}^{(3)}}=\frac{1}{6} \frac{S f(\Pi)}{\operatorname{Vol}(\Pi)} .
$$

From mean value relations that can be derived from the mean values calculated in the last sections we obtain furthermore

$$
\begin{aligned}
& L_{K_{1}}=\frac{1}{12} \frac{S f(\Pi)}{\operatorname{Vol}(\Pi)}, \quad L_{J_{1}^{(2)}}=\frac{3}{28} \frac{S f(\Pi)}{\operatorname{Vol}(\Pi)}, \\
& L_{I_{1}}=\frac{1}{4} \frac{S f(\Pi)}{\operatorname{Vol}(\Pi)}, \quad U_{K_{2}}=\frac{3}{7} \frac{S f(\Pi)}{\operatorname{Vol}(\Pi)}, \\
& U_{J_{2}}=\frac{2}{3} \frac{S f(\Pi)}{\operatorname{Vol}(\Pi)}, \quad U_{I_{2}}=\frac{S f(\Pi)}{\operatorname{Vol}(\Pi)}, \\
& A_{K_{2}}=\frac{2}{7} \frac{b r(\Pi)}{\operatorname{Vol}(\Pi)}, \quad A_{I_{2}}=2 \frac{b r(\Pi)}{\operatorname{Vol}(\Pi)} .
\end{aligned}
$$

Similarly, the corresponding intensities can be expressed in terms of $\mathrm{Vol}$. For example we have $\lambda_{V_{T}}=4 \operatorname{Vol}(\Pi), \lambda_{V_{C}}=2 \operatorname{Vol}(\Pi)$ and $\lambda_{V}=\lambda_{V_{T}}+\lambda_{V_{X}}=$ $6 \operatorname{Vol}(\Pi)$.
It is an important observation that any of the metric mean values from above is a constant times $S f(\Pi) / \operatorname{Vol}(\Pi)$ or $\operatorname{br}(\Pi) / \operatorname{Vol}(\Pi)$. These expressions are well known from convex geometry as isoperimetric and the latter as isepiphanic coefficient. By applying classical inequalities for intrinsic volumes of convex bodies from convex geometry, see for example Gruber (2007), we can deduce that the mean values $L_{Y_{1}}$, $U_{X_{2}}$ and $A_{X_{2}}$ with $Y \in\left\{K, J^{(2)}, J^{(2)}, I\right\}$ and $X \in$ $\{I, J, K\}$ achieve their minimal values if and only if the associated Steiner compact is a ball. This is exactly the case, when the directional distribution $\mathcal{R}$ is the uniform distribution on $\mathbb{S}_{+}^{2}$, i.e., in the case when the homogeneous random STIT tessellation is additionally isotropic. The lower bounds for the resulting inequalities can now be obtained from the the previous section and we get the following couple of inequalities:

$$
\begin{aligned}
& L_{K_{1}} \geq \frac{1}{S_{\mathcal{V}}}, \quad L_{J_{1}^{(2)}} \geq \frac{9}{7 S_{\mathcal{V}}}, \quad L_{J_{1}^{(3)}} \geq \frac{2}{S_{\mathcal{V}}}, L_{I_{1}} \geq \frac{3}{S_{\mathcal{V}}} \\
& U_{K_{2}} \geq \frac{36}{7 S_{\mathcal{V}}}, \quad U_{J_{2}} \geq \frac{8}{S_{\mathcal{V}}}, \quad U_{I_{2}} \geq \frac{12}{S_{\mathcal{V}}}, \\
& A_{K_{2}} \geq \frac{48}{7 \pi S_{\mathcal{V}}^{2}}, A_{J_{2}} \geq \frac{16}{\pi S_{\mathcal{V}}^{2}}, A_{I_{2}} \geq \frac{48}{\pi S_{\mathcal{V}}},
\end{aligned}
$$

with equality holding only in the isotropic case.

\section{ACKNOWLEDGMENTS}

The authors would like to thank Claudia Redenbach (Kaiserslautern) for providing the two beautiful simulations of the STIT and the one of the Poisson plane tessellation. The first author was supported by the Swiss National Science Foundation Grant SNF PP002-114715/1.

\section{REFERENCES}

Gruber P (2007). Convex and discrete geometry. Berlin: Springer.

Mackisack M, Miles R (1996). Homogeneous rectangular tessellations. Adv Appl Probab 28:993-1013.

Matheron G (1975). Random sets and integral geometry. Chichester: Wiley.

Mecke J, Nagel W, Weiss V (2007). Length distributions of edges in planar stationary and isotropic STIT tessellations. J Contemp Math Anal 42:28-43.

Mecke J, Nagel W, Weiss V (2008a). A global construction of homogeneous random planar tessellations that are stable under iteration. Stochastics 80:51-67.

Mecke J, Nagel W, Weiss V (2008b). The iteration of random tessellations and a construction of a homogeneous process of cell divisions. Adv Appl Probab 40:49-59. 
Mecke J (2009). Joint distribution of direction and length of the typical $I$-segment in a homogeneous random planar tessellation stable under iteration. J Contemp Math Anal 44:45-53.

Mecke J, Nagel W, Weiss V (2010). Some distributions for $I$-segments of planar random homogeneous STIT tessellations. Math Nachr 284:1483-95.

Nagel W, Weiss V (2003). Limits of sequences of stationary planar tessellations. Adv Appl Probab 35:123-38.

Nagel W, Weiss V (2005). Crack STIT tessellations - characterization of stationary random tessellations stable with respect to iteration. Adv Appl Probab 37:859-83.

Nagel W, Weiss V (2006). STIT tessellations in the plane.
Rend Circ Matem Palermo II, Suppl. 77:441-58.

Nagel W, Weiss V (2008). Mean values for homogeneous STIT tessellations in 3D. Image Anal Stereol 27:29-37.

Schneider R, Weil W (2008). Stochastic and integral geometry. Berlin: Springer.

Schreiber T, Thäle C (2010). Typical geometry, secondorder properties and central limit theory for iteration stable tessellations. arXiv:1001.0990 [math.PR].

Stoyan D, Kendall WS, Mecke J (1995). Stochastic geometry and its applications. 2nd Ed. Chichester: Wiley.

Thäle C (2009). Moments of the length of line segments in homogeneous planar STIT tessellations. Image Anal Stereol 28:69-76. 